\title{
Low-Temperature Mobility of Surface Electrons and Ripplon-Phonon Interaction in Liquid Helium
}

\author{
A. I. Safonov ${ }^{1)}$, I. I. Safonova and S.S. Demukh \\ Russian Research Centre Kurchatov Institute, pl. Akademika Kurchatova 1, Moscow, 123182 Russia \\ Submitted February 4, 2010 \\ Resubmitted March 4, 2010
}

\begin{abstract}
The low-temperature dc mobility of the two-dimensional electron system localized above the surface of superfluid helium is determined by the slowest stage of the longitudinal momentum transfer to the bulk liquid, namely, by the interaction of surface and volume excitations of liquid helium, which rapidly decreases with temperature. Thus, the temperature dependence of the low-frequency mobility is $\mu_{\mathrm{dc}} \approx 8.4 \times$ $10^{-11} n_{e} T^{-20 / 3} \mathrm{~cm}^{4} \mathrm{~K}^{20 / 3} /(\mathrm{V} \cdot \mathrm{s})$, where $n_{e}$ is the surface electron density. The relation $T^{20 / 3} E_{\perp}^{-3} \ll 2 \times 10^{-7}$ between the pressing electric field (in $\mathrm{kV} / \mathrm{cm}$ ) and temperature (in $\mathrm{K}$ ) and the value $\omega \lesssim 10^{8} \cdot T^{5} \mathrm{~K}^{-5} \mathrm{~s}^{-1}$ of the driving-field frequency have been obtained, at which the above effect can be observed. In particular, $E_{\perp} \simeq 1 \mathrm{kV} / \mathrm{cm}$ corresponds to $T \lesssim 70 \mathrm{mK}$ and $\omega / 2 \pi \lesssim 30 \mathrm{~Hz}$.
\end{abstract}

'PACS: $05.60 . \mathrm{Gg}, 67.25 . \mathrm{du}, 68.03 . \mathrm{Kn}$, 73.20.Qt

Low-dimensional systems of charges have been 'studied theoretically and experimentally for about four decades. These systems include the two-dimensional electron gas in semiconductor structures and the electron systems localized above the surface of liquid helium [1, 2]. The relaxation times of the excited spin or Rydberg states of surface electrons turn out to be much longer owing to a weak coupling to the helium surface. This makes the surface electrons (SE) quite attractive as possible memory units of a quantum computer 3. At the same time, the relative weakness of the relaxation mechanisms determines the ultimately 'high mobility $\mu \sim 10^{4} \mathrm{~m} / \mathrm{V} \cdot \mathrm{s}$ of the SEs, probably the highest of all known two-dimensional systems.

At $T<0.7 \mathrm{~K}$, when the saturation pressure of helium vapor is exponentially small, the SE mobility is limited by their interaction with ripplons, the elementary excitations of the helium surface, whereas the direct electron-phonon interaction is ineffective. All previous theoretical and experimental works on the SE mobility assumed that the longitudinal momentum 'of the electrons transferred due to their interaction with ripplons is immediately accumulated by the entire liquid (see, e.g., [1. However, there are indications that in fact the relaxation of the longitudinal momentum of the electrons proceeds in two stages, via the momentum transfer to ripplons and further from the ripplons to the phonons of the bulk liquid, and is limited by the slowest stage.

Generally speaking the experimental observation of the ripplon-phonon interaction is a separate and quite

\footnotetext{
1) safonov@isssph.kiae.ru
}

formidable problem. One of possible solutions is to measure the heat transfer along the helium surface [4]. However, in this case, one has to use thin films to avoid a giant-effective thermal conductance of a bulk liquid, which could easily mask the surface heat transfer. On the other hand, in the case of films, an important role is played by the surface smoothness, as the ripplons can be scattered not merely by phonons but also by surface imperfections caused by substrate defects (roughness). Such a one-body elastic scattering of ripplons has a much weaker temperature dependence than the ripplonphonon interaction and therefore dominates at low temperature [5]. Guaranteed exclusion of this one-body elastic channel of the ripplon flow dissipation imposes quite severe requirements on the surface smoothness over a large area. Thus, the contribution of the ripplon-phonon processes to the thermal conductance of helium surface turns out to be barely detectable. Roche et al. 6] implemented capacitive excitation and detection to measure the damping of long-wavelength ripplons. However, this technique is hardly applicably for studying thermal ripplons whose wavelength is just $10 \mathrm{~nm}$, i.e., three orders of magnitude shorter than in experiments of Roche et al. In this work, we show that the use of surface electrons might be very promising.

The surface (ripplons) and volume (phonons) quasiparticles of liquid helium interact only to the extent of a small compressibility of helium. The rate of the dominant process of momentum transfer at low temperatures (the phonon creation with the annihilation of two ripplons [7]) is proportional to $T^{20 / 3}[5]$. Consequently, the ripplon subsystem in the 
$T \rightarrow 0$ limit appears to be practically isolated from the liquid bulk and has to follow the regular motion of the electrons. Thus, the SE mobility should infinitely increase with a decrease in temperature. However, nothing of that kind was observed in experiments at least down to $20 \mathrm{mK}$ [8, 9]. On the contrary, the mobility of the electrons, which form a Wigner solid [10, 11] at low temperature, even somewhat decreases and levels off at $T \rightarrow 0$. This work is aimed at resolving this contradiction, finding the conditions, under which the SE mobility is determined by the ripplon-phonon interaction (or, at least, when the respective contribution is considerable), and calculating the dependence of the mobility on temperature, the frequency $\omega$ of the driving electric field $E_{\|}(t)=E_{\|} \mathrm{e}^{i \omega t}$ and the magnitude of the pressing field $E_{\perp}$.

We suggest that the said contradiction is caused by the fact that the measurements are usually conducted in the ac regime at a relatively high frequency and that the electron effective mass is several orders of magnitude less than that of ripplons. As a result, the amplitude of the in-plane oscillations of the ripplons subsystem is vanishingly small. A mechanical analogue of this situation could be a small rubber on the surface of a massive plate, which in turn lies on a smooth ice. The in-plane motion of the rubber driven by a periodic force is detected. It is intuitively clear that in order to observe the joint motion of electrons and ripplons relative to the bulk liquid one has to perform the measurements in the dc regime or at a very small frequency. It is also clear that one should aim at increasing the electron-to-ripplon ratio of the effective mass densities.

We shall consider the ripplon and electron subsystems separately. This requires that the equilibration time within each subsystem be substantially shorter than the relaxation time between the subsystems. Next, we do not include the effect of the transverse magnetic field, which is typically used in the mobility measurement in the Corbino geometry but absolutely insignificant for the present problem. The amplitude $E_{\|}$of the driving electric field is thought to be sufficiently small so that the effects associated with the electron overheating could be neglected. We also disregard the boundary effects assuming the infiniteness of the two-dimensional system. Taking into account the above remarks, the equations of motion of electrons and ripplons in the $\tau$ approximation read as

$$
\frac{d \mathbf{u}_{e}}{d t}=-\frac{e \mathbf{E}_{\|}}{m^{*}}+\frac{\mathbf{u}_{\mathrm{R}}-\mathbf{u}_{e}}{\tau_{e}}
$$

and

$$
\frac{d \mathbf{u}_{\mathrm{R}}}{d t}=-\gamma \frac{\mathbf{u}_{\mathrm{R}}-\mathbf{u}_{e}}{\tau_{e}}-\frac{\mathbf{u}_{\mathrm{R}}}{\tau_{\mathrm{RP}}},
$$

respectively. Here, $\mathbf{u}_{e}\left(\mathbf{u}_{\mathrm{R}}\right)$ is the in-plane velocity of the electron (ripplon) subsystem as a whole, $e$ is the unit charge, $m^{*}$ and $n_{e}$ are the the effective mass and number density of electrons, $\rho_{\mathrm{R}}=1.67 \times 10^{-10} T^{5 / 3} \mathrm{~K}^{-5 / 3} \mathrm{~g} / \mathrm{cm}^{2}$ is the mass density associated with ripplons, $\gamma=$ $n_{e} m^{*} / \rho_{\mathrm{R}}$ is the electron-to-ripplon mass-density ratio. The electron momentum-relaxation time $\tau_{e}$ is related to the SE mobility $\mu$ with respect to ripplons as $\tau_{e}=$ $m^{*} \mu / e$ (see below) and weakly depends on temperature in the range under consideration. According to the experimental data on the electron mobility [12 and effective mass [13, the electron-ripplon relaxation time at $T=70 \div 80 \mathrm{mK}$ and $n_{s}=1.3 \times 10^{9} \mathrm{~cm}^{-2}$ is $\tau_{e} \sim 5 \times 10^{-7} \mathrm{~s}$. Thus, at $T \lesssim 0.1 \mathrm{~K}$ the ripplon-phonon momentum relaxation time $\tau_{\mathrm{RP}}=8.8 \times 10^{-9} T^{-5} \mathrm{~K}^{5} \mathrm{~s}[5$ is much greater than $\tau_{e}$. Note, that the comprehensive kinetic calculation of $\tau_{e}$ for free electrons is also based on the equilibrium of the electron and ripplon subsystem 1]. In particular, according to [1, the rate of electronelectron collisions in a typical experiment is much higher than $\tau_{e}^{-1}$.

In a weak pressing field $E_{\perp}$, the effective mass of electrons almost coincides with their bare mass, $m^{*} \approx$ $m$. In this case, the electrons appear to be orders of magnitude "lighter" than the ripplons $(\gamma \ll 1)$ and the latter do not feel the electron motion, $u_{\mathrm{R}} \ll u_{e}$. In this case, Eq. (1) yields the usual expression for the electron mobility (to be compared with Eq. (10.7) of [1])

$$
\mu \equiv-\frac{\operatorname{Re} u_{e}}{E_{\|}}=\left(\frac{e}{m^{*}}\right) \frac{\tau_{e}}{1+\omega^{2} \tau_{e}^{2}} .
$$

At not to high driving-field frequency $\omega \tau_{e} \ll 1$ (which is equivalent to neglecting the left-hand side of Eq. (11)), we find $\mu=e \tau_{e} / m^{*}$ [14.

In a strong pressing field the picture is substantially changed. First of all, the maximum achievable electron density increases proportional to the pressing field, $E_{\perp}=2 \pi e n_{e}^{\max }$. In addition, the electron effective mass as such increases by two or even three orders of magnitude. In particular, $m^{*} / m$ increases by from 500 to 5000 nearly proportional to the square of the pressing field $E_{\perp}=0.57 \div 1.672 \mathrm{kV} / \mathrm{cm}$ at a constant density $n_{e}=6.3 \times 10^{8} \mathrm{~cm}^{-2}$ [15] and slightly decreases (from 2000 to 1500 ) with an increase in density from 5 to $12 \times 10^{8} \mathrm{~cm}^{-2}$ at a constant pressing field $E_{\perp}=1.15 \mathrm{kV} / \mathrm{cm}$ [13. An increase in the effective mass is caused by the fact that the strong field presses electrons into the helium surface, thus forming dimples. Therefore, the electron motion along the surface involves a considerable mass of helium associated with the displacement of the dimples. Though the resultant mass densities of electrons and ripplons are still substantially 
different, this mass increase may be sufficient to fulfill the condition $\tau_{e} \ll \gamma \tau_{\mathrm{RP}}$, which is equivalent to $\mu / e n_{e} \ll \tau_{\mathrm{RP}} / \rho_{\mathrm{R}} \simeq 53 T^{-20 / 3} \mathrm{~K}^{20 / 3} \mathrm{~s} \cdot \mathrm{cm}^{2} / \mathrm{g}$. For example, at $T=70 \mathrm{mK}, n_{e}=10^{9} \mathrm{~cm}^{-2}$ and $E_{\perp}=$ $1 \mathrm{kV} / \mathrm{cm}$ one has $\tau_{e} \simeq 5 \times 10^{-7} \mathrm{~s}, \gamma \simeq 3 \times 10^{-3}$, $\tau_{\mathrm{RP}} \simeq 5 \times 10^{-3} \mathrm{~s}$ and, consequently, $\gamma \tau_{\mathrm{RP}} \sim 1.5 \times 10^{-5} \mathrm{~s}$. In this case, we find from Eq. (2) with $\omega \tau_{e} \ll 1$ that the electron and ripplon velocities almost coincide, $u_{\mathrm{R}} \approx u_{e} \equiv u$, and, consequently,

$$
u \approx-e E_{\|} \frac{n_{e} \tau_{\mathrm{RP}}}{\rho_{\mathrm{R}}}\left(\frac{1+i \omega \tau_{\mathrm{RP}}}{1+\omega^{2} \tau_{\mathrm{RP}}^{2}}\right),
$$

where we neglected $\gamma$ with respect to unity.

As expected, the amplitude $|u|$ of the velocity oscillations has a maximum at zero frequency of the driving field and decreases as $1 / \omega$ at high frequencies $\left(\omega \tau_{\mathrm{RP}} \gg 1\right)$ with a $\pi / 2$ phase lag compared to the driving field. The appearance of the out-of-phase component of the electron velocity (and, consequently, the current) serves as the direct evidence of the discussed effect. This component has a maximum at $\omega \tau_{\mathrm{RP}}=1$, which is exactly one half of the maximum value of the velocity. Equation (4) with $\omega=0$ yields the dc electron mobility relative to the bulk liquid

$$
\mu_{\mathrm{dc}}=\frac{e n_{e} \tau_{\mathrm{RP}}}{\rho_{\mathrm{R}}} \approx 8.4 \times 10^{-11} n_{e} T^{-20 / 3} \frac{\mathrm{cm}^{4} \mathrm{~K}^{20 / 3}}{\mathrm{~V} \cdot \mathrm{s}} .
$$

The found temperature dependence of the lowfrequency mobility for $n_{e}=1.4 \times 10^{7}, 1.08 \times 10^{8}$ and $1.3 \times 10^{9} \mathrm{~cm}^{-2}\left(E_{\perp}=1.25 \mathrm{kV} / \mathrm{cm}\right)$ is shown in figure by the dotted, dashed and solid line, respectively. The results of mobility measurements via the longitudinal ac conductivity $\sigma_{x x}$ of the two-dimensional system in the Corbino geometry at $\omega / 2 \pi=10 \mathrm{kHz}, E_{\perp}=15 \mathrm{~V} / \mathrm{cm}, n_{e}=1.4 \times 10^{7} \mathrm{~cm}^{-2}[9]$; $\omega / 2 \pi=10 \mathrm{kHz}, E_{\perp}=92.5 \mathrm{~V} / \mathrm{cm}, n_{e}=1.08 \times 10^{8} \mathrm{~cm}^{-2}$ 8, and via the damping of the mixed phonon-ripplon modes of a Wigner solid at $\omega / 2 \pi=5 \div 10 \mathrm{MHz}$, $n_{e}=1.3 \times 10^{9} \mathrm{~cm}^{-2}[12]$ are also shown for comparison.

The theoretical dc mobility of free electrons relative to ripplons in a strong pressing field is [16]

$$
\mu_{0}=\frac{8 \alpha \hbar}{e^{2} E_{\perp}^{2}} \frac{e}{m}=\frac{9.3 \times 10^{11} \mathrm{~V} / \mathrm{s}}{E_{\perp}^{2}},
$$

where $\alpha=0.378 \mathrm{erg} / \mathrm{cm}^{2}$ is the surface tension of helium. Thus, in full agreement with experiments [17. 18. 19, the mobility is inversely proportional to the square of the pressing field and does not depend on temperature. In the case of a Wigner solid, the behavior of the mobility changes substantially, first of all, because, depending on the magnitude $E_{\|}$and $E_{\perp}$

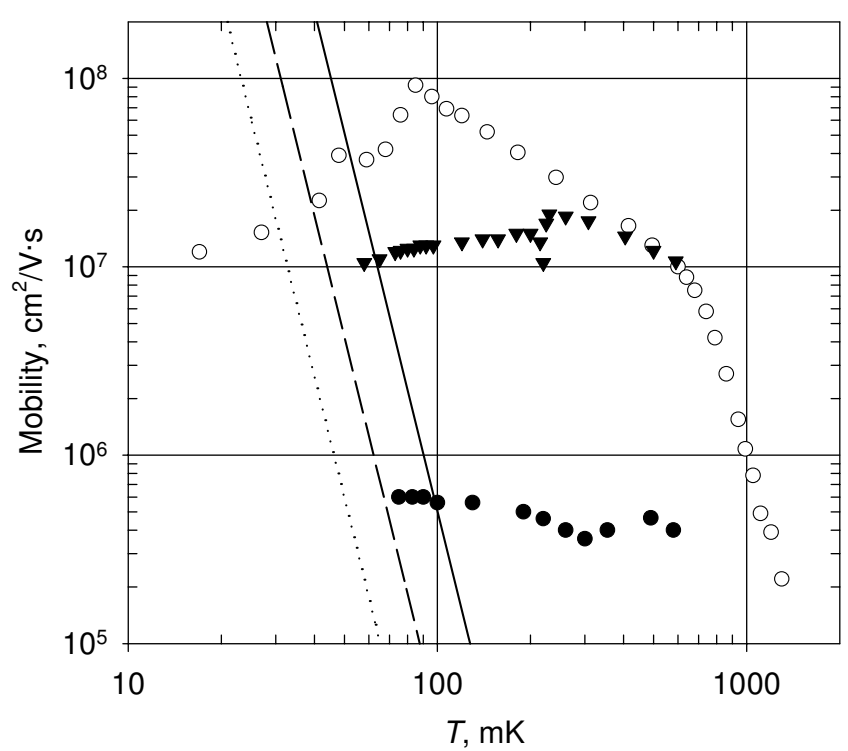

Temperature dependence of the SE mobility measured via the longitudinal ac conductivity $\sigma_{x x}$ of the twodimensional system in the Corbino geometry at (o) $\omega / 2 \pi=10 \mathrm{kHz}, E_{\perp}=15 \mathrm{~V} / \mathrm{cm}, n_{e}=1.4 \times 10^{7} \mathrm{~cm}^{-2}$ 9]; $(\mathbf{\nabla}) \omega / 2 \pi=10 \mathrm{kHz}, E_{\perp}=92.5 \mathrm{~V} / \mathrm{cm}, n_{e}=$ $1.08 \times 10^{8} \mathrm{~cm}^{-2} 8$, and $(\bullet)$ via the damping of the mixed phonon-ripplon modes of a Wigner solid at $\omega / 2 \pi=5 \div 10 \mathrm{MHz}, n_{e}=1.3 \times 10^{9} \mathrm{~cm}^{-2} 12$. The straight lines show the dc mobility (5) for the respective values of $n_{e}$ (from left to right in the order of increasing density). Some experimental points are omitted for better visibility.

of the pressing and driving field, excitation frequency and temperature, the two-dimensional electron system may find itself in a so-called coupled phonon-ripplon (CPR) state formed by the electrons and the respective dimples on the helium surface or slide over the helium surface [20, 21]. Under the transition between these two states, the electron mobility exhibits an abrupt change, which may reach an order of magnitude. In the CPR state of interest, which in fact appears in strong pressing fields and a weak driving field and at low frequencies, the dependence $\mu \propto E_{\perp}^{-2}$ is generally preserved [15, 22] and the mobility of a Wigner solid also weakly depends on temperature [9, 12, 15]. Taking for the estimate the value $\mu=6 \times 10^{5} \mathrm{~cm}^{2} / \mathrm{V} \cdot \mathrm{s}$ obtained at $n_{e}=1.3 \times 10^{9} \mathrm{~cm}^{-2}$ and $T=70 \mathrm{mK}$ [12, we find that the rates of momentum transfer from electrons to ripplons and from ripplons to photons of helium become equal at $T \simeq 98 \mathrm{mK}$ (see figure). For the ripplon-phonon interaction to be the bottleneck of the longitudinal momentum relaxation the measurements should be carried out at $T \lesssim 70 \mathrm{mK}$. According to Eq. (44), the frequency $\omega$ of the driving field in this case 
should not be greater than $\tau_{\mathrm{RP}}^{-1} \simeq 1.1 \times 10^{8} T^{5} \mathrm{~K}^{-5} \mathrm{~S}^{-1}$, which corresponds to $\omega / 2 \pi \lesssim 30 \mathrm{~Hz}$ at the specified temperature.

Thus, we have seen that observing the ripplonphonon interaction in liquid helium via the SE mobility requires the simultaneous fulfillment of the conditions

$$
\tau_{e} \ll \frac{m^{*} n_{e}}{\rho_{\mathrm{R}}} \tau_{\mathrm{RP}}
$$

and

$$
\omega \tau_{\mathrm{RP}} \lesssim 1
$$

Taking into account the dependence of the effective mass $m^{*}$ and limiting density $n_{e}$ of the surface electrons on the pressing field $E_{\perp}$ and the temperature dependence of $\rho_{\mathrm{R}}$ and $\tau_{\mathrm{RP}}$, these conditions may be rewritten in a more practical form

$$
\begin{gathered}
T^{20 / 3} E_{\perp}^{-3} \ll 2 \times 10^{-7}, \\
\omega / 2 \pi \lesssim 1.5 \times 10^{7} T^{5} \mathrm{~Hz},
\end{gathered}
$$

where the temperature, field and frequency are expressed in Kelvin, $\mathrm{kV} / \mathrm{cm}$ and $\mathrm{Hz}$, respectively.

In experiments of Dotsenko et al. [12, the condition (9) was fulfilled. However, the condition (10) was violated so that the relative contribution of the ripplonphonon interaction in helium to the mobility of the electron crystal was no more than $\sim 10^{-5}$. The technique of mobility measurement via the damping of hybrid phonon-ripplon modes of a Wigner solid seems to be fundamentally inapplicable for observing the effect under consideration, since the minimum frequency of the phonon-ripplon modes is given by the lattice constant of the electron crystal and the dispersion relation of ripplons and therefore cannot be smaller than $\sim(\alpha / \rho)^{1 / 2} n_{e}^{3 / 4}$, where $\rho=0.145 \mathrm{~g} / \mathrm{cm}^{3}$ is the mass density of ${ }^{4} \mathrm{He}$ at $T=0223$. The mobility of the two-dimensional electron system can be also determined from its longitudinal conductivity $\sigma_{x x}$ measured in the Corbino geometry. However, the respective experiments [8, 9] violated both conditions, primarily because of the smallness of the pressing field. In particular, at $E_{\perp}=$ $92.5 \mathrm{~V} / \mathrm{cm}$ the inequalities (9), (10) yield $T \leq 25 \mathrm{mK}$ and $\omega / 2 \pi \lesssim 0.14 \mathrm{~Hz}$ whereas the measurements were carried out at 10 and $100 \mathrm{kHz}$.

Thus, we have shown that the ripplon-phonon interaction in superfluid helium can be studied with the use of surface electrons and determined the conditions to be satisfied by the experimental parameters, i.e., temperature, excitation frequency, the density of the two-dimensional electron system, and the magnitude of the pressing electric field.
We are truly grateful to S.S.Sokolov and V.E.Sivokon' for fruitful discussions, to K.Kono for providing us with some of his relevant publications and to I.S.Yasnikov for reading the manuscript and valuable remarks. This work was supported by the Russian Foundation for Basic Research, project no. 09-02-01002-a.

Список литературы

1. V. B. Shikin and Yu. P Monarkha, Two-Dimensional Charged Systems in Helium (Moscow, Nauka, 1989) (in russian).

2. V. S. Edelman, Sov. Phys. Uspekhi 23, 227 (1980) [Usp. Fiz. Nauk. 130, 675 (1980)].

3. M. I. Dykman, P. M. Platzman and P. Seddighrad, Phys. Rev. B 67, 155402 (2003).

4. I. B. Mantz, D. O. Edwards and V. U. Nayak, Phys. Rev. Lett. 44, 66 (1980); Errata 44, 1094 (1980).

5. A. I. Safonov, S. S. Demukh and A. A. Kharitonov, JETP Lett. 79, 304 (2004) [Pis'ma Zh. Eksp. Teor. Fiz. 79, 362 (2004)].

6. P. Roche, G. Deville, K. O. Keshishev, N. J. Appleyard, and F. I. B. Williams, Phys. Rev. Lett. 75, 3316 (1995).

7. M. W. Reynolds, I. D. Setija and G. V. Shlyapnikov, Phys. Rev. B 46, 575 (1992).

8. K. Shirahama, S. Ito, H. Suto, and K. Kono, J. Low Temp. Phys. 101, 439 (1995).

9. K. Kono and K. Shirahama, in Two-Dimensional Electron Systems, ed. E.Y.Andrei (Kluwer Acad. Publ., 1997), p.p. 175-189.

10. E. Wigner, Trans. Faraday Soc. 34, 678 (1938).

11. C. C. Grims and G. Adams, Phys. Rev. Lett. 42, 795 (1979).

12. V. V. Dotsenko, V. E. Sivokon', Yu. Z. Kovdrya, and V. N. GrigorЎev, Low Temp. Phys. 23, 772 (1997) [Fiz. Nizk. Temp. 23, 1028 (1997)].

13. V. E. Syvokon, Yu. Z. Kovdrya, and K. A. Nasyedkin, J. Low Temp. Phys. 144, 35 (2006).

14. We disregard a factor-of-two difference in $\tau_{e}$ at high- and low-frequency excitation 18 because of the approximate character of the inequality (7), which includes $\tau_{e}$.

15. M. A. Stan and A. J. Dahm, Phys. Rev. B 40, 8995 (1989)

16. V. B. Shikin and Yu. P. Monarkha, J. Low Temp. Phys. 16, 193 (1974).

17. C. C. Grims and G. Adams, Phys. Rev. Lett. 36, 145 (1976).

18. P. M. Platzman and G. Beni, Phys. Rev. Lett. 36, 626 (1976).

19. B. N. Esel'son, A. S. Rybalko and S. S. Sokolov, Sov. J. Low Temp. Phys. 6, 544 (1980) [Fiz. Nizk. Temp. 6, 1120 (1980)]. 
20. K. Shirahama and K. Kono, Phys. Rev. Lett. 74, 781 (1995).

21. K. Kono and K. Shirahama, Surf. Sci. 361/362, 826 (1996).

22. R. Mehrotra, C. J. Guo, Y. Z. Ruan, D. B. Mast, and A. J. Dahm, Phys. Rev. B 29, 5239 (1984).

23. G. Deville, J. Low. Temp. Phys. 72, 135 (1988). 